Revue

d'ethnoécologie
Revue d'ethnoécologie

$6 \mid 2014$

Conservation de la nature : quel rôle pour les sciences sociales?

\title{
La gestion participative des forêts en Afrique centrale
}

Logique développementiste, gouvernance antiparticipative et stratégies d'acteurs

Participative management of the forests in Central Africa : developmentist logic, antiparticipative governance and actors strategies

Daou Véronique Joiris, Patrice Bigombe Logo et Séverin Cécile Abega (†)

\section{(2) OpenEdition}

Journals

Édition électronique

URL : https://journals.openedition.org/ethnoecologie/1960

DOI : $10.4000 /$ ethnoecologie. 1960

ISSN : 2267-2419

Éditeur

Laboratoire Éco-anthropologie

Référence électronique

Daou Véronique Joiris, Patrice Bigombe Logo et Séverin Cécile Abega ( + ), « La gestion participative des forêts en Afrique centrale ", Revue d'ethnoécologie [En ligne], 6 | 2014, mis en ligne le 31 décembre 2014, consulté le 07 décembre 2022. URL : http://journals.openedition.org/ethnoecologie/1960 ; DOI : https://doi.org/10.4000/ethnoecologie.1960

Ce document a été généré automatiquement le 29 septembre 2020.

Creative Commons - Attribution - Pas d'Utilisation Commerciale - Pas de Modification 4.0 International - CC BY-NC-ND 4.0

https://creativecommons.org/licenses/by-nc-nd/4.0/ 


\title{
La gestion participative des forêts en Afrique centrale
}

\author{
Logique développementiste, gouvernance antiparticipative et stratégies \\ d'acteurs $^{1}$ \\ Participative management of the forests in Central Africa : developmentist logic, \\ antiparticipative governance and actors strategies
}

Daou Véronique Joiris, Patrice Bigombe Logo et Séverin Cécile Abega ( $†$ )

À la mémoire du regretté Séverin Cécile Abéga, décédé inopinément le 24 mars 2008 à Yaoundé, au Cameroun.

\section{La gestion participative des forêts : un modèle à l'épreuve de la réalité}

1 Dans une synthèse sur l'anthropologie du développement international, Mosse (2013) évoquant Participation: The new tyrany? (Cooke \& Kothari 2001) et Participation: From tyrany to transformation? (Hickey \& Mohan 2004) ${ }^{2}$ rappelle que l'approche participative est analysée plutôt comme structurée par des relations de pouvoir que comme modifiant celles-ci. Ces politiques, écrit Mosse, peuvent favoriser à la fois une forme de « recontextualisation techniciste $\|^{3}$ des " savoirs et des institutions locales » et une forme « d'engendrement de modes de vie et d'aspirations novatrices", "mettant en place de nouvelles échelles de distinction sociale, mais laissant de larges structures non altérées » (Mosse 2013: 229) ${ }^{4}$. Le développement peut donc être envisagé comme une « catégorie de pratique ", un modèle à l'épreuve de la réalité.

2 Des campagnes équato-guinéennes et gabonaises aux campagnes camerounaises, centrafricaines et congolaises, ce n'est un secret pour personne, les régions touchées par les réformes participatives n'ont pas fondamentalement décollé économiquement. La documentation fine dont fait état le présent article collectif $^{5}$ porte sur cinq des 
mécanismes participatifs expérimentés par des agences de développement en appui à des instances nationales et à des ONG de divers horizons (rente forestière annuelle communautaire, rente de superficie, forêt communautaire, zone d'intérêt cynégétique à gestion communautaire, activités de conservation et de développement intégrées). Elle conforte les écrits précédents en soulignant sans détour les faibles retombées pour le développement rural. C'est là un constat amer, particulièrement pour des régions nanties sur les plans financier, écologique et climatique mais où les besoins sociaux sont critiques.

3 L'analyse des retombées sociales du mécanisme de la redevance forestière annuelle réalisée par Mbetoumou et al. (2010 : 57-81) porte sur les 783 millions de francs CFA (71 millions d'euros) de l'exercice budgétaire 2005, soit près de 10 millions de francs CFA par village et un peu plus de 2 millions de francs CFA par tranche budgétaire ${ }^{6}$. D'autres montants, comparativement aussi colossaux si l'on se réfère aux économies familiales, sont repris par Karsenty (2010: 35-53) sur le régime concessionnaire, par Bigombe Logo et al. (2010:105-119) sur la foresterie sociale ainsi que par Bigombe Logo \& Roulet (2010: 143-151) sur la gestion communautaire des zones de chasse. (Voir également Cerutti et al. 2010a, 2010b ; Assembe 2004).

4 Les raisons de l'échec de la gestion participative sont complexes. Elles sont structurelles et politiques. Nos recherches comparatives dans les secteurs de l'exploitation industrielle du bois, de la chasse sportive, de la gestion des aires protégées, des forêts et des zones de chasse communautaires montrent que les conditions dans lesquelles les populations "bénéficiaires" de ces politiques sont gouvernées s'inscrivent dans des continuités historiques colonialistes, néo- et post colonialistes difficilement en phase avec les intentions et les ambitions égalitaristes et vertueuses de l'approche participative. Les difficultés rencontrées par le modèle participatif sont d'abord structurelles, c'est-à-dire, liées à l'histoire et à la configuration des sociétés humaines qui accueillent les dispositifs participatifs. Elles sont ensuite conjoncturelles et contextuelles. Elles relèvent, à cet effet, des logiques et stratégies d'acteurs à l'égard des mécanismes participatifs particuliers. Les détournements, les contournements, les appropriations et les rejets sont mus par des logiques diverses, mais qui ont pour point commun d'être étrangères à la logique développementaliste qui sous-tend les réformes réalisées. Ce deuxième niveau d'analyse des politiques « participatives » renvoie au cas de figure, bien connu et bien étudié par la sociologie des organisations et la socio-anthropologie du développement, de la confrontation entre le mode de gouvernance et la façon dont celui-ci est approprié et capturé par les destinataires.

5 L'article met d'abord en évidence le caractère immuable du concept de "participation » tel que véhiculé au sein de l'univers professionnel développementiste qualifié par Apthorpe (2011 : 210-211) d'history nullius. Il aborde ensuite les causes socio-politiques et historiques puis conjoncturelles des difficultés de mise en œuvre de cette approche en se situant dans la perspective « actors oriented» initiée par Long (voir Long \& Long 1992). La conclusion présente la nécessité du monitoring des pratiques empiriques de la gestion participative et du développement rural effectif.

\section{Une amnésie développementiste}

6 De nombreuses études attestent des résultats mitigés de la gestion participative telle qu'appliquée depuis une trentaine d'années dans le Bassin congolais (Cuny 2011; Ezzine 
de Blas et al. 2011, 2009; Garcia \& Lescuyer 2008; Cheka 2007; Julve et al. 2007 ; Ngo Youmba 2007; Oyono et al. 2007a; Lescuyer 2006; Karsenty 2006; Nasi et al. 2006; Nguinguiri \& Pierre 2006; Oyono 2006, 2005a, 2005b; Oyono \& Efoua 2006; Oyono et al. 2006a, 2006b; Vermeulen et al. 2006; Diaw 2005; Bigombe Logo 2004; Cuny et al. 2004 ; Bigombe Logo 2003b ; Etoungou 2003 ; Compagnon \& Constantin 2000 ; Lavigne Delville et al. 2000 ; Diaw et al. 1998; IIED 1994 ; Weber 2000 - pour ne citer que ces auteurs); et ailleurs dans le monde (Sundström 2013; Sunderland et al. 2012; Larson et al. 2010; Sommerville et al. 2010 ; Sletto 2009 ; Ribot 2008, 2004a, 2004b ; Meral et al. 2008 ; Nelson \& Agrawal 2008; Igoe \& Brockington 2007 ; Tacconi 2007 ; De Jong et al. 2006 ; Iversen et al. 2006 ; Colfer \& Capistrano 2005 ; Larson 2005 ; Pacheco 2005 ; Siswanto \& Wardojo 2005 ; Kumar 2002; Li 2002; Rodary 2001; Robbins 2000; Turner 1999; Uphoff 1998; Wollenberg 1998 ; Wells et al. 1992 ; Kiss 1990 ; Lavigne Delville 1992). Une revue de cette abondante littérature montre que les politiques de gestion participative ont généré des revenus considérables. Des sommes importantes ont été détournées et les infrastructures construites sont inopérationnelles alors que l'offre participative a des effets négatifs pour certaines catégories de la population et que d'autres nouvelles démarches développementistes héritées de la décentralisation, de la privatisation et de la néolibéralisation s'avèrent aussi peu performantes (voir par exemple Rajak 2011; Ferguson 2010, 2006, 2005 ; Diop 2006 ; Ribot et al. 2006 ; Graig \& Porter 2006 ; Ferguson \& Gupta 2002).

7 Si la faiblesse du décollage économique participatif des zones rurales est alarmante, l'occultation de ce constat par les décideurs mérite également d'être soulignée. On relève au sein des institutions de développement une forte disparité entre le discours sur les bienfaits de la participation et la pratique participative qui n'atteint pas les objectifs assignés. On relève de surcroît le déni des implications négatives de cette politique et on peut pointer la capacité du champ développementiste à reconduire et reproduire la disparité entre le discours et la pratique plutôt que d'y apporter des mesures de correction. Pourquoi cette lacune institutionnelle ne fait-elle pas l'objet de remaniements compte tenu de la mise en œuvre tâtonnante de la participation? Et par quels mécanismes se reproduit-elle?

Dans le fonctionnement développementiste, la dénégation de la marge entre les intentions et les réalisations est bien connue. Celle-ci a été interprétée comme un moyen de préserver la politique en tant que structure de représentation (Mitchell 2002, cité par Mosse 2013 : 13) et comme expression de relations de pouvoirs (Li 2007 ; Ferguson 1994). Dans le Bassin congolais, certains aspects organisationnels du secteur professionnel participent de cet état de choses : le désintérêt pour les réalisations de terrain, l'absence de monitoring des logiques et stratégies d'acteurs vis-à-vis de l'offre effective de participation, les manquements en termes de suivi, de contrôle et de reddition des comptes (accountability), le laisser-faire, la coordination lacunaire des interventions, le maintien du mode de fonctionnement autoritaire en dépit de la proclamation de l'adhésion à la participation, etc. Il n'existe pas à proprement parler d'évaluations empiriques présentant un bilan chiffré de façon exhaustive. La mesure de l'impact de ces politiques à l'échelle locale, du point de vue de la gestion financière, de la gestion administrative et de la maitrise d'ouvrage pour les équipements d'intérêt public, ne semble pas faire partie de l'agenda des décideurs. On peut également noter un manque d'harmonisation des stratégies de développement relié à la démarche participative dans 
son ensemble. Ce qui soulève le problème supplémentaire d'incohérence d'une telle politique.

9 Ainsi que le rappellent Sunderland et al. (2012 : 421) citant Garnett et al. (2007) ; Sayer et al. (2007) ainsi que Kapos et al. (2009) concernant des bilans relatifs au modèle ICDP ${ }^{7}$ :

« [...] les projets de conservation sont en général pauvrement suivis et évalués [...] malgré une pléthore d'écrits sur les méthodologies, [...] malgré le consensus au sein des chercheurs, des bailleurs, des conservationnistes et des agences de développement que le suivi et l'évaluation sont nécessaires pour apprendre et adapter [la politique]. Il y a une reconnaissance croissante que l'information anecdotique provenant des projets [...] doit être remplacée par une solide base d'informations qui puisse guider les gestionnaires des projets et ultimement informer les décideurs. Cependant, la preuve est répandue d'un échec ou d'une faible performance des monitoring $"$.

10 Dans ce contexte, la propension à l'autocritique et à la régulière redéfinition du concept participatif au sein du discours développementiste sont des traits spécifiques à faire ressortir. Le lexique relatif à la dimension techniciste de la participation est riche, dense, foisonnant. La rhétorique participative, comme celle de l'ensemble du champ développementiste, véhicule des concepts au sens littéral de « représentations générales et abstraites de la réalité », des "western fantaisies » (Kupper 2003), des "travelling concepts » (Tsing 1999) , des «travelling rationalities » (Mosse 2011b), désignés par un lexique technique présentant le trait commun à l'ensemble des agences de développement d'être polysémique et flou. De prime abord, on en retire l'impression de repositionnements en fonction des interfaces avec le terrain. L'évolution lexicale ressort de multiples ateliers et rencontres entre décideurs, agents de terrain, représentants des communautés, ainsi que de l'amélioration fréquente des outils participatifs. La littérature sur la gestion participative environnementale, c'est-à-dire appliquée à la gestion des ressources naturelles, est imposante. Elle puise pour une large part dans le registre de l'appui techniciste à la mise en œuvre des mécanismes participatifs, à la promotion de méthodes d'enquêtes participatives, de consultation populaire, de gestion des conflits, et à la valorisation des savoirs écologiques traditionnels. L'autre registre saillant est celui de l'analyse plus ou moins critique des mécanismes participatifs mis en œuvre sur le terrain. La constitution de comités de gestion et l'élaboration de plans de développement local ont fait l'objet de toutes les attentions de la part des professionnels qui ont élaboré diverses Méthodes accélérées de recherche participative (Marp), du diagnostic Rural rapide appraisal (Rra) à l'Apprentissage participatif et recherche actions (Apra). Des expériences de cogestion de la nature ont mobilisé les sciences sociales et ont été relayées au niveau des concepteurs de ces programmes (voir par exemple Lockwood et al. 2010; Oyono et al. 2007b ; Béné \& Neiland 2006 ; Bigombe Logo 2002, 2003a ; Emerit \& Lescuyer 2003 ; Borrini-Feyerabend et al. 2004 ; Uphoff 1998 ; Wade 1988). Pourtant, quand on y prête attention, le caractère dynamique de la rhétorique participative opère comme un écran de fumée, un affichage. À titre d'exemple, on observe, dans la gestion de la redevance forestière annuelle au Cameroun, un décalage entre les intentions juridiques vertueuses de l'arrêté conjoint nº 076 MINATD/MINFI/MINFOF du 26 juin 2012 fixant les modalités de planification, d'emploi et de suivi de la gestion des revenus provenant de l'exploitation des ressources forestières et fauniques destinés aux communes et aux communautés villageoises riveraines et son application effective sur le terrain. L'affichage camouffle la superficialité des révisions. Le vocabulaire est renouvelé alors que la politique et sa pratique ne le sont pas. Le remaniement du lexique s'applique en réalité à un modèle pratique inchangé, la participation étant invariablement conçue 
comme une démarche démocratique et égalitaire s'adressant à des communautés projetées comme démocratiques et égalitaires ${ }^{8}$ ainsi qu'à des contextes socio-politicohistoriques intrinsèquement compatibles avec cette démarche ${ }^{9}$. Étant donné l'incapacité au sein du champ à considérer les nombreux constats faisant état de récurrents rapports de force communautaires à l'interface avec le contexte, étant donné donc l'incapacité à modifier l'approche participative en fonction de ceux-ci, on peut parler sans exagération d'une forme d'amnésie structurelle en tant que cadre social de la mémoire (Douglas 1986, 2004 : 106) telle que traitée dans des études spécifiques à l'institution développementiste, c'est-à-dire, l'attitude qui consiste à oublier les échecs et/ou les succès connus dans les approches et les actions de développement menées dans le passé et à reprendre ou refaire les mêmes choses d'un cycle de développement à un autre (Joiris \& Bigombe Logo 2012; Apthorpe 2011; Birschenk 2009; Lewis 2009; Rossi 2007; Tsing 1999; Isaacman 1997; Wade 1996).

11 Dans le Bassin congolais comme ailleurs, définir à quoi correspond l'approche «participative» relève d'une gageure tant l'expression, devenue commune dans la rhétorique du développement, recouvre une multitude de pratiques et de niveaux d'implication des populations dans la réalisation concrète du développement local. Ces pratiques et niveaux d'implication renvoient à diverses modalités relationnelles qui vont d'une simple information des populations à, beaucoup plus rarement, une réelle implication de ces dernières dans les prises de décision. Si l'« approche participative » est en ce sens polysémique et recouvre des degrés variables d'implication, l'analyse doit également englober des situations qualifiées de participatives mais qui, en réalité, n'ont rien de participatif. Dans les faits, l'«approche participative» ne correspond jamais, premièrement, à la participation de l'ensemble des couches de la population et, deuxièmement, à la cogestion effective du développement local.

\section{Un mode de gouvernance antiparticipatif}

12 D'après la bibliographie de notre étude, les retombées sociales de politiques fiscales et territoriales décentralisées expérimentées depuis une trentaine d'années dans le Bassin congolais sont faibles. Cela soulève des questions sur la pertinence du mode de gouvernance mis en œuvre.

La seule expérience participative relativement durable, celle de la Compagnie équatoriale des bois (CEB) au Gabon (Nguimbi et al. 2010: 86-102), s'inscrit plutôt dans la responsabilité sociale et environnementale, le volet social de la certification forestière appliqué par une dizaine de compagnies forestières pionnières.

La prise en compte du contexte historique et sociopolitique d'implantation des politiques participatives permet d'approcher une mesure, à l'échelle locale, de l'impact de ces politiques. L'analyse comparative des cinq mécanismes participatifs cités, tous secteurs confondus, met en évidence une série de points communs. Malgré d'apparentes différences, les principes de gestion sont similaires et ont été mis en place dans des contextes historiques eux aussi similaires où le régime concessionnaire joue un rôle décisif.

15 L'analyse croisée, dans une perspective historique, du contexte d'implantation des mécanismes participatifs dans les secteurs du bois, de la faune et des aires protégées, 
révèle dans quelle mesure la gouvernance participative est conditionnée par le dispositif financier, territorial et juridique adopté dans les pays d'Afrique centrale.

Compte tenu des déterminants historiques et sociopolitiques de la sous-région, le contexte d'implantation de la gouvernance participative est à la fois néocolonial et non démocratique; des relations très marquées de patronage et de clientélisme y prévalent. Structurellement parlant, il présente des incompatibilités avec la participation attendue. Selon nous, la base structurelle de la gestion participative explique pour une large part les résultats mitigés. Elle a trois caractéristiques récurrentes qu'il nous paraît important de souligner : un mode de fonctionnement top-down et dirigiste, un dispositif territorial source d'insécurité foncière et un cadre juridique lacunaire.

\section{Un fonctionnement top-down et dirigiste}

17 La contribution financière au développement local "participatif » dans le Bassin congolais s'inscrit dans un cadre qui reste foncièrement néocolonial et paternaliste malgré la mise en place d'un dispositif extrêmement novateur du point de vue de la responsabilisation des populations locales.

Le montage financier " participatif » repose essentiellement sur la fiscalité décentralisée. Des sommes colossales sont rétrocédées aux communes et aux communautés villageoises riveraines. Au Cameroun, par exemple, de l'année 2000 à 2007, une somme globale de 47 milliards de francs CFA a été rétrocédée de l'État aux communes et communautés villageoises riveraines des forêts soumises à l'exploitation forestière industrielle. Pourtant, cette approche se révèle inefficace dans la réalisation du développement au regard des détournements dont souffre la filière. Les montants dégagés par la fiscalité décentralisée sont les plus importants, mais ils sont détournés par des élites rurales et régionales qui ont tendance à se constituer en « entrepreneurs de la gouvernance locale des forêts et en « écopouvoir » local » et en interlocuteurs incontournables.

Par ailleurs, les revenus financiers tirés de la gestion des forêts communautaires et des territoires de chasse communautaire ne semblent pas encore suffisants pour promouvoir des réalisations tangibles et durables de développement local. Dans certains cas, les revenus issus des forêts communautaires et des territoires de chasse communautaire sont faibles par rapport aux besoins et aux attentes des populations.

En réalité, les résultats les plus probants sont essentiellement du ressort du secteur privé, d'initiatives à connotation communautaire (redevance de superficie, obligations sociales), mais qui ont pour caractéristique d'être gérées par l'entrepreneur privé dans un partenariat direct avec certaines franges des populations locales, sans impliquer les représentants des ministères de tutelle et de l'administration déconcentrée (mairie), ni les chefferies traditionnelles.

21 Toutefois, ainsi que le soulignent Nguimbi et al. (2010: 86-102) dans « Une gestion locale assumée par un opérateur privé", les dispositions "participatives» de l'exploitant consignées dans les cahiers des charges et dans les plans d'aménagement représentent vraisemblablement les apports les plus constants et les plus opérationnels. C'est d'autant plus vrai que l'exploitant a développé un savoir-faire dans la réalisation d'infrastructures sociales au profit des populations locales. Ce succès s'explique en grande partie par la maitrise, de bout en bout, du processus par le concessionnaire. 

développement au suivi des dépenses et des réalisations sur le terrain. Il évite ainsi habilement certains dérapages de la part des élites et des populations elles-mêmes. Cependant, c'est de fait un mode de gouvernance dirigiste, de type top-down, aux relents colonialistes. Les privés, généralement expatriés, exercent souvent leur profession dans un contexte rappelant celui du patronat des débuts de l'industrialisation européenne et des concessions forestières coloniales (Karsenty 2010 : 35-55). Ce mode de gouvernance produit pratiquement les seuls résultats tangibles et d'envergure en matière de participation. Du point de vue idéologique, il s'agit donc d'un mode de gouvernance dirigiste et antiparticipatif.

zones de chasse dite villageoise, dont le modèle participatif a été initié dans les années 1990, dans le nord de la République centrafricaine (Roulet \& Hardin 2010 : 123-139; Bigombe Logo \& Roulet 2010: 143-151), les protocoles d'accord entre le gestionnaire de la zone de chasse, les représentants de l'administration et les populations comportent des règles coercitives vis-à-vis des populations. Et les mêmes espaces font l'objet d'une exploitation depuis la période coloniale. Les principes appliqués ne sont qu'une actualisation des procédures colonialistes antérieures. La pratique «participative» dans les safaris comporte une dimension raciste bien ancrée dans les mentalités des opérateurs privés et de leurs clients. Elle relève de ce fait d'un paradigme antagoniste par rapport aux valeurs participatives.

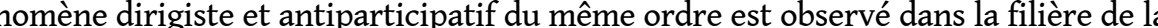
rente forestière et des terres à vocation communautaire où ce sont les élites qui prennent généralement les initiatives. Les plans de développement dans lesquels sont consignées des orientations théoriquement choisies par les populations présentent la caractéristique courante, voire commune, de promouvoir un développement local standardisé. Les financements dégagés débouchent, par exemple, sur des salles de classe ou des dispensaires construits à l'identique le long des pistes d'une circonscription.

Ces orientations sont, pour la plupart, définies "d'en haut », par la mairie et des élites politiciennes. Elles aboutissent à un développement de surface (les bâtiments restent souvent inachevés et inoccupés), suivant un souci de visibilité électoraliste de la part des décideurs locaux. Comme le montrent nos études de cas sur la redevance forestière annuelle (RFA) communautaire et sur les forêts communautaires au Cameroun, les autorités locales font en général peu appel à la consultation populaire afin d'identifier les besoins réels des populations.

financement du développement local, à savoir la configuration développementaliste classique (les projets de développement et les ONG), est caractérisée par un manque de coordination entre bailleurs de fonds, ainsi qu'entre ces derniers et les politiques nationales et, a fortiori, entre les différentes filières de financement du développement local participatif. Ce manque de coordination se traduit par la superposition des initiatives, des chevauchements et des doublons dans les actions de développement réalisées. Les projets ne remplissent pas les conditions d'une réelle implication de la société civile. Ils ont certes une assise communautaire, mais le point d'impact se situe souvent à un niveau «local» qui n'englobe pas les instances décisionnelles villageoises et communales.

Le comité villageois promu par ce modèle de développement est une création exogène qui double, sans l'englober, le conseil villageois où siègent la chefferie et des notables. Ce 
refus de reconnaître les capacités villageoises à gérer la chose publique paraît paradoxal au vu des efforts déployés par ailleurs pour susciter une démarche participative. Par exemple, depuis l'institution de la redevance forestière annuelle, la part destinée aux communautés villageoises riveraines est toujours versée dans les comptes financiers des communes et gérée par les maires en lieu et place des institutions villageoises. Le mode de gouvernance participatif mis en œuvre par la coopération au développement présente ainsi les stigmates d'une trentaine d'années durant lesquelles les interventions de l'État et des bailleurs de fonds internationaux ont été principalement fondées sur de grands programmes de développement conçus au niveau central, et d'une cinquantaine d'années d'application de modèles de développement occidentalo-centristes.

À l'évidence, les continuités historico-politiques particulières dans lesquelles s'inscrivent les dispositifs financiers associés à la décentralisation du développement local expliquent le caractère dirigiste et antiparticipatif de ce mode de gouvernance. Les deux modes de financement les plus récents, à savoir la fiscalité décentralisée via le niveau intermédiaire de l'administration décentralisée et des comités villageois, d'une part, et les terres à vocation communautaire, d'autre part, semblent être appliqués au sein de l'histoire particulière de la relation entre les élites politiciennes et affairistes et les populations. En effet, la redistribution des taxes par l'administration décentralisée reproduit au niveau villageois ce que les politologues appellent «le modèle étatique de prédation » ou le «mimétisme rentier».

\section{Un dispositif territorial source d'insécurité foncière}

Le rapport entre la décentralisation et le développement local dans lequel s'inscrit l'approche participative peut également être envisagé sous l'angle des questions foncières et territoriales. Bien que constituant un des bastions du développement local, ces questions sont globalement passées sous silence dans les analyses actuelles.

Le zonage d'application dans le Bassin congolais montre clairement que la part la plus étendue du territoire forestier est attribuée aux exploitations forestières et aux aires protégées, les franges restantes étant dévolues à d'autres usages tels les safaris et aux populations rurales. Il faut aussi mentionner les dynamiques actuelles de superposition des activités minières, des agro-industries et des exploitations forestières. La superficie relative des espaces d'après le zonage indique que les terres octroyées en concession par l'État à des privés occupent la première place. Ensuite viennent les terres mises en réserve, puis les terres que l'État met à la disposition des communautés locales et, en dernier lieu, les finages villageois.

31 Ces terres peuvent se superposer et font l'objet d'une exploitation par rotation. Le découpage foncier et territorial inhérent à de telles stratégies de développement local intègre seulement de façon partielle les espaces d'activités économiques des populations. Pour ce qui est du zonage appliqué dans le Bassin congolais, certains parlent de plan d'exclusion des populations locales. Si l'on se réfère à des études ethnoécologiques (par exemple, Dounias 1993 et 2000), toutes proportions gardées, la superficie octroyée par le zonage aux finages villageois est insignifiante au regard des surfaces nécessaires au bon fonctionnement écologique et socio-économique des systèmes d'exploitation en vigueur en forêts denses et humides. 
32 La partition du territoire reflète la priorité donnée à la vocation commerciale selon un mode économique de rentabilité capitaliste qui laisse très peu de place aux modes communautaires d'exploitation forestière. Une continuation, en quelque sorte, du "paradigme forestier colonial» qui met en avant la rentabilité financière des écosystèmes avant leur utilité sociale et culturelle. En milieu forestier, l'étendue des finages est pourtant vaste à l'échelle d'un village. Elle dépasse largement les espaces dévolus aux activités locales d'après le zonage. En réalité, la délimitation actuelle des finages villageois est une délimitation par défaut. Elle résulte d'un recouvrement par d'autres types d'espace et se situe en bordure de piste, en dehors des concessions dont l'implantation est antérieure à la réforme participative, réglementairement selon les pays, sur une bande de trois, cinq ou dix kilomètres de part et d'autre de la piste.

Ces zones villageoises ne sont pas reconnues en tant que telles par l'aménagiste puisque amputées d'une large part de leur superficie. Elles ne font l'objet d'aucun projet d'aménagement particulier en dehors de celui qui consiste à en limiter de facto l'expansion, contrairement aux concessions forestières ou de chasse dont les modalités d'exploitation sont consignées dans des plans simples de gestion.

Notre analyse de l'évolution historique du régime concessionnaire (Karsenty 2010 : 35-53) et celle des concessions de chasse (Roulet \& Hardin $2010: 123-139)$ et des aires protégées (Binot et al. 2010 : 155-175) éclaire cette difficulté à intégrer les finages villageois dans les plans de zonage. Les dispositions actuelles s'inscrivent dans le prolongement d'un aménagement du territoire datant de la fin du $\mathrm{XIX}^{\mathrm{e}}$ siècle qui consacrait le non-respect de ce que l'on appelait à l'époque les « réserves indigènes ».

La contribution de Karsenty (2010: 35-53) souligne l'ambivalence qui a existé dès le départ entre la volonté de voir les sociétés concessionnaires n'exploiter que les terres situées en dehors des villages (zones indigènes de culture, de pâturage ou forestières) et la volonté d'éviter la destruction des forêts.

L'idée de la "concession de conservation » lancée ces dernières années par de grandes organisations de conservation ne fait que s'inscrire dans cette continuité historique. Elle s'appuie en effet sur des mécanismes de mise en conservation de nouvelles portions de finages et implique le versement d'une rente monétaire aux populations et aux États en compensation du renoncement des différents acteurs à leurs activités habituelles comme l'exploitation ou la chasse.

37 Contrairement à ce que l'on pourrait imaginer de prime abord, l'établissement de terres à vocation communautaire a également pour effet d'accroître les contraintes sur les droits d'usage directs des populations locales. Roulet et Hardin (2010) indiquent à ce propos que la reproduction de dispositions ségrégationnistes, exclusives, ainsi que la confiscation de terres qui sont observées dans les zones de chasse, s'inscrivent dans la foulée des pratiques coloniales et conduisent à ce qu'on peut appeler la « permanence des entraves à une réappropriation ».

38 Notre étude documente les recouvrements territoriaux générés par l'approche participative non seulement dans les aires protégées, mais également dans le domaine de la redevance de superficie et dans les terres à vocation communautaire. Les conflits dans la gestion de forêts communautaires (Bigombe Logo et al. 2010 : 105-119) résultent en partie de la cession d'espaces fonciers et forestiers coutumiers au profit du développement de la collectivité, ce qui donne lieu à des revendications de compensations. 
39 Ainsi, du point de vue du dispositif territorial et à l'inverse de ce que la rhétorique de la cartographie participative pourrait laisser croire, on constate une impossibilité politique et historique à négocier ou à concilier les points de vue sur la circonscription des espaces par rapport aux finages villageois. Ces finages sont tout simplement absents des considérations de l'aménagiste. La partition du territoire en espaces à vocation économique spécialisée prévaut dans toute démarche "participative »; elle est imposée de fait par des dispositions légales et techniciennes, occidentalo-centristes et néolibérales, héritées du régime concessionnaire de la fin du XIX ${ }^{e}$ siècle.

Un paradoxe majeur est que le politique reconnait des pertes de terres et de ressources par un dispositif financier compensatoire et l'octroi de terres à vocation communautaire, tout en niant la part la plus importante des pertes puisque des pans entiers des finages villageois sont amputés. Dans les aires protégées, où les politiques dites participatives sont les plus anciennes, le paradoxe est à son comble puisque aucun dédommagement n'est envisagé pour compenser les pertes de terres, de cultures et de ressources occasionnées par l'implantation des zones de conservation.

41 Quel que soit le cas de figure, l'absence de planification pour la gestion des finages villageois est préoccupante non seulement du point de vue de la reproductibilité des systèmes économiques locaux, mais également du point de vue de leur durabilité écologique.

\section{Un cadre juridique insuffisamment élaboré}

42 Ces deux dernières décennies, les pays du Bassin congolais ont adopté de nouvelles législations forestières et foncières favorables à la décentralisation. Ces cadres législatifs garantissent certains droits aux populations, mais présentent encore de nombreuses imprécisions, particulièrement concernant les procédures de recours pour les résidents locaux. Le dispositif juridique qui garantit le développement rural dans les contextes de décentralisation et de participation spécifiques des pays concernés se révèle insuffisant et inefficace au regard des nombreux défis liés aux remaniements en cours.

Ce dispositif révèle un problème de fond: la reconnaissance rhétorique du droit coutumier est en porte-à-faux avec le cadre légal moderne pour lequel le droit coutumier est inexistant sauf, dans une certaine mesure, au Congo et en République démocratique du Congo, où des aspects de droit coutumier sont intégrés dans le droit forestier positif. Dans la plupart des cas, les populations ne peuvent recourir au code coutumier en cas de litiges " participatifs».

44 Les observateurs s'accordent sur la faiblesse du dispositif juridique qui sous-tend la politique "participative» et la décentralisation dans son ensemble. Les problèmes les plus saillants sont la non-obligation de rendre des comptes aux populations, qui constitue un obstacle au bon fonctionnement des comités de gestion, l'absence de valeur légale attribuée aux entités participatives et aux conventions entre parties prenantes, ainsi que la non prise en compte de l'appropriation coutumière des terres et des forêts.

D'après les nouvelles législations forestières et les plans d'aménagement, le niveau de reconnaissance des espaces dévolus aux populations est faible et varie selon le statut foncier et territorial. Ces espaces ne sont en aucune manière des propriétés communautaires. Un bon indicateur du faible degré de sécurisation foncière du dispositif d'aménagement $\mathrm{du}$ territoire en tant que moyen de promouvoir «l'approche 
participative » est l'inexistence de procédés d'expropriation et de compensation tels que pratiqués dans les pays du Nord.

Le cas de figure plus ou moins sécurisant est la forêt communautaire qui équivaut à des ressources que l'État accorde en concession aux communautés locales organisées en entités juridiques reconnues. Au Cameroun, la forêt communautaire correspond à une variante ancrée dans le territoire, mais sous tutelle de l'administration, et non des populations elles-mêmes. D'autres terres ont finalement un statut local précaire et circonstanciel. Le statut des zones à exploitation villageoise, à la périphérie des aires protégées, n'est « activé » que durant la période de réalisation des activités de gestion de l'aire protégée, théoriquement par un plan d'aménagement, mais rares sont les plans d'aménagement opérationnels, et par un cadre législatif national harmonisé par rapport à des normes internationales.

Enfin, le fait de reconnaître l'existence des finages et de les mesurer afin de calculer une redistribution fiscale (redevance de superficie) ou de moduler les obligations sociales des cahiers des charges en fonction de l'implantation territoriale des riverains ne signifie pas pour autant que des droits d'usage et un droit de propriété soient octroyés officiellement.

\section{Des enjeux locaux récurrents autour du mode de gouvernance antiparticipatif}

48 L'étude des logiques et stratégies des acteurs vis-à-vis du mode de gouvernance participative permet d'approcher, de façon complémentaire, la mesure de l'impact local des politiques de gestion participative. L'analyse transversale et comparative des cinq mécanismes participatifs met en lumière une autre série de points communs. Ce sont des logiques et des stratégies similaires du point de vue "endogène » qui peuvent être identifiées quel que soit le mécanisme participatif - fiscalité décentralisée, attribution de droits de gestion ou implication dans des activités de développement et de conservation intégrées.

49 Partout, le mode de gouvernance participative induit des enjeux locaux fonciers, d'accès aux ressources et de représentativité, ainsi que des équilibrages dans les communautés villageoises entre les personnes qui s'approprient le processus et celles qui ne réussissent pas à se l'approprier, soit entre dominants et dominés.

50 Il entraîne un accès inégal à la terre et à ses ressources, des captations financières, matérielles et symboliques à géométrie variable, ainsi que des enjeux de représentativité locale traversés par des rapports de patronage et de sorcellerie. Ce sont là des entraves conjoncturelles qu'il est possible d'analyser, d'autant qu'elles sont récurrentes.

51 L'approche "participative » telle que proposée aux populations, quel que soit le type de financement, d'espace ou de cadre juridique, fait l'objet d'enjeux récurrents autour desquels les acteurs mobilisent des logiques et stratégies. Qu'il s'agisse de la RFA communautaire, de la rente de superficie, des forêts et des zones de chasse communautaires ou d'activités de conservation et de développement intégrées, ces mécanismes induisent partout des conflits relativement importants dans les communautés rurales.

52 Ces conflits sont l'expression de tensions très profondes qui ont pour caractéristiques d'être latentes et difficilement compréhensibles de prime abord, et de relever d'enjeux 
liés au mode de gouvernance participatif. Étant donné la récurrence de ces enjeux dans l'ensemble des mécanismes participatifs investigués, tous secteurs confondus, nous proposons une grille d'analyse qui consiste à les regrouper dans trois principaux domaines : l'accès à la terre et à ses ressources, l'accès aux apports financiers, en nature et symboliques des mécanismes participatifs et l'accès à la représentativité dans les instances participatives comme les comités de gestion.

\section{Un accès inégal à la terre et à ses ressources}

Les pertes de terres occasionnées par le dispositif territorial dans lequel s'inscrit l'approche participative sont un enjeu majeur pour la plupart des paysans, parce qu'ils doivent se repositionner afin de conserver l'accès aux ressources naturelles. Le redéploiement territorial actuel des populations en réaction aux restrictions d'accès instaurées par le zonage est globalement passé sous silence dans la littérature. En revanche, celle-ci est très fournie concernant les espaces à vocation communautaire qui ne représentent pourtant qu'une faible portion des finages villageois.

Les dynamiques territoriales locales en réaction au mode de gouvernance "participatif » font l'objet de peu d'analyses pour deux raisons: d'une part, un manque de volonté politique et, d'autre part, une faible mobilisation locale, de nombreuses tensions territoriales étant en quelque sorte occultées. Comme le soulignent Roulet \& Hardin (2010 : 123-139), le point de vue des populations se manifeste sous forme de "rancune » ou de " déresponsabilisation " plutôt que de revendications formelles.

Binot et al. (2010: 155-175) soulignent par ailleurs que la pluralité des interventions inhérentes aux processus de décentralisation en cours induit les utilisateurs des ressources naturelles en erreur sur leurs droits d'accès et d'usage, sur l'autorité foncière et sur les mécanismes de régulation des conflits. La position subalterne des paysans dans l'arène participative, aux côtés des représentants de l'administration, des opérateurs privés et des ONG, n'autorise peut-être pas une forme plus "politisée » des réactions locales vis-à-vis des restrictions d'accès induites par le mode de gouvernance " participatif ». On pourrait de surcroît considérer que l'opacité est renforcée du fait que les peuples de la forêt expriment peu leur identité territoriale, leurs revendications n'étant pas nécessairement ancrées dans le territoire.

L'identité spatiale se manifeste seulement lorsque l'accès à la terre et à ses ressources occasionne des rapports compétitifs entre usagers locaux. Les conflits territoriaux, même s'ils augmentent avec le temps, sont rares, ponctuels, les forêts étant peu peuplées et s'étendant sur de vastes superficies. En effet, l'exploitation industrielle n'est perceptible que sporadiquement dans les zones d'usage villageois, en fonction des modalités d'extraction et la plupart des paysans ignorent souvent dans quelles mesures «leurs » forêts se trouvent dans une concession forestière ou de chasse.

57 Afin de mieux approcher la sensibilité locale vis-à-vis des questions foncières et territoriales, il est nécessaire de faire une distinction entre les finages villageois tels qu'ils existaient avant le zonage - que nous pourrions qualifier d'anciennes terres villageoises et les terres à vocation communautaire créées par la démarche "participative ». Les terres à vocation communautaire mises à disposition des communautés locales correspondent ainsi à de nouvelles terres villageoises qui se superposent aux anciennes sans nécessairement les englober totalement. 

exemple, planches, tôles ou routes) ou symboliques telles qu'une meilleure assise sociale, statutaire et politicienne, des relations ou des protections. Deux aspects, trop peu abordés dans la littérature, méritent l'attention des décideurs à ce niveau. Premièrement, le mode de gouvernance "participatif» implique la modification des systèmes de production économique endogène et ce changement ne débouche pas, tout au moins dans l'immédiat, sur une augmentation généralisée des revenus en milieu rural.

Deuxièmement, le modèle de développement est appliqué dans un contexte socioéconomique qui combine de façon dialectique deux types d'économies, l'économie de marché et l'économie communautaire, au sens défini par Gudeman (2001), de sorte que 
les résidents locaux ne réagissent pas d'après le seul modèle de l'économie néolibérale qui est intégré dans le mode de gouvernance participative. L'enjeu est de taille. Il s'agit, pour les paysans les plus démunis, de s'adapter à de nouvelles situations et, pour d'autres, de se positionner afin d'en valoriser les opportunités.

Comme pour les questions territoriales, cet enjeu est à l'origine de stratégies locales relativement « invisibles », qui ne sont pas toujours portées explicitement par les acteurs eux-mêmes et qui ne font donc pas nécessairement l'objet de revendications, d'autant que les effets de la réforme participative ne seront perceptibles qu'à moyen ou long terme.

Toute introduction de nouvelles modalités d'exploitation des ressources naturelles occasionne des changements dans les modes de production. La modification des superficies, des plantes et des animaux élevés ou prélevés, des quantités ainsi que des techniques, a des effets sur les agencements entre producteurs et sur les modes de production. Contrairement à l'idéalisation rhétorique des savoirs écologiques traditionnels, la gouvernance participative, du point de vue des paysans, équivaut au passage de savoir-faire locaux à de nouvelles modalités d'exploitation. Le zonage et les plans d'aménagement maintiennent ou déséquilibrent les modes de production indigènes en remaniant la superficie, la qualité et la quantité des ressources naturelles ainsi que l'accès à la terre.

Les activités de développement ont très souvent pour ambition d'accroître la production des espèces cultivées sur des superficies restreintes en lieu et place du prélèvement de ressources sauvages sur de vastes superficies. Il s'agit d'un mouvement d'occidentalisation et d'introduction de nouvelles modalités d'exploitation des écosystèmes qui, à la fois, accompagne et conditionne les financements du développement local «participatif ». Selon la perception des populations locales, la mise en place de cette politique va de pair avec une période d'adaptation et de transition et des processus plus ou moins lents selon les catégories d'acteurs, processus allant de l'incapacité de s'adapter à l'appropriation.

Du point de vue endogène, l'accès à de nouvelles terres villageoises nécessite l'adoption de nouvelles modalités d'exploitation. Ce passage est et restera pendant plusieurs générations lent et différencié selon le capital économico-culturel des groupes locaux. Vues sous cet angle, les retombées du mode de gouvernance " participatif » en termes de lutte contre la pauvreté sont largement différées et potentiellement inéquitables. N'oublions pas que des changements structurels comme ceux ambitionnés par les concepteurs des mécanismes participatifs prennent en général bien plus qu'une génération à moins de se dérouler dans un contexte révolutionnaire ou autoritaire.

L'ampleur de la réforme participative proposée dans le Bassin du Congo est considérable en termes de développement rural. Or, elle contraste fortement avec la quasi-absence de planification, de contrôle et de suivi de la part des décideurs, ce qui renvoie à un paradoxe supplémentaire inhérent au mode de gouvernance " participatif ».

En l'absence d'un contrôle extérieur, les comptes ne sont pas transparents et la reconstitution des dépenses est laborieuse. En effet, les dépenses sont orientées tant vers des priorités sociales que vers des priorités matérielles et les décaissements de prestige occupent une place importante dans les caisses familiales. Dès lors, nombre de sorties sous forme "d'enveloppes» tendent à satisfaire des besoins d'ordre statutaire, relationnel et politicien, ce qui ne rentre pas dans les objectifs du développement local «participatif». 


\section{De la représentativité locale aux rapports de patronage}

70 Le dysfonctionnement des comités de gestion est une constante, quel que soit le mécanisme participatif envisagé. Qu'il s'agisse de gestion de terres à vocation communautaire, de partenariats avec des exploitants privés ou d'organisation des populations à la périphérie des aires protégées, le conseil villageois est très peu ou pas du tout représentatif de l'ensemble des habitants d'un village ou d'une région.

71 Confortant des études antérieures, nos recherches rapportent que les comités de gestion sont dirigés pour l'essentiel par des élites intérieures et extérieures à la communauté villageoise, exception faite $d u$ partenariat impliquant la CEB au Gabon. Ce dysfonctionnement paraît inhérent aux difficultés de mise en œuvre de la gouvernance participative. On le retrouve dans d'autres contextes de décentralisation que celui de l'Afrique centrale (Ribot 2008, 2004a, 2004b).

Il n'en reste pas moins que ce déficit de démocratie verte est enraciné dans des contextes historico-sociaux particuliers, ce qui plaiderait pour la prise en compte d'éléments conjoncturels complémentaires, parmi lesquels le patronage et la sorcellerie qui semblent très présents dans le Bassin congolais.

Nos études de cas, à l'appui d'autres publications (comme celles de Ngo Youmba 2007 ; Nelson \& Agrawal 2008) montrent que la gestion décentralisée des forêts est caractérisée par des accointances entre élites et lignages ou clans dominants et que les enjeux de représentativité des communautés locales dans les instances participatives donnent lieu à des stratégies ascendantes et descendantes articulées sur des rapports clientélistes et de domination très présents.

74 Ce système est marqué par ce que certains qualifient de "culture politique paroissiale " et Sindjoun (1998) de "politique d'affection » sous-tendues par des processus identitaires et xénophobes (Geschiere 2004). Dans un contexte politique traditionnel décentralisé, les individus ignorent le système politique global et se tournent vers des sous-systèmes politiques plus limités (lignages, clans). C'est donc à une échelle que l'on pourrait qualifier d'infra-locale, en tout cas en dessous de l'échelle d'intervention du mode de gouvernance participatif, que s'opèrent les recentrages locaux vis-à-vis de cette politique.

Les rapports de clientélisme et de domination omniprésents sur le terrain s'inscrivent en partie dans la continuité de relations dites de patronage qui remontent aux civilisations protobantoues précoloniales (Vansina 1991). On retrouve les figures du patron et du client dans la plupart de nos études de cas, particulièrement dans l'analyse des logiques et stratégies d'acteurs vis-à-vis des concessions forestières. L'analyse de Karsenty (2010: 35-53) semble rejoindre celle sur la relation entre populations et gestionnaires d'une aire protégée, présentée par Solly (2010: 177-193). La position de patron implique des abus potentiels; le conflit, la tension et le manque de confiance caractérisent la position du client et constituent finalement le principal frein à l'exercice d'une gestion vraiment participative. On est ainsi loin d'une appropriation par les populations sur un mode participatif, puisque le gestionnaire est conçu comme un patron, de surcroît corrompu et assimilé à l'État, donc dominant les communautés locales.

76 Mbetoumou et al. (2010: 57-81) relèvent, quant à eux, l'existence de relations de domination de l'ordre du clientélisme politique doublées de relations de l'ordre de la parenté (oncle maternel et neveu utérin). Bigombe Logo et al. (2010: 105-119) font le 
même constat concernant les forêts communautaires : il y a mobilisation des clientèles et des parentèles en vue d'obtenir un pouvoir dans les entités juridiques et ainsi accéder aux revenus des forêts.

Dans l'arène participative, cette coconstruction des relations entre intervenants sur l'archétype du patron et du client semble largement contribuer aux nombreuses incompréhensions relevées entre populations et projets participatifs.

Dans le contexte particulier des sociétés segmentaires et à la faveur des liens d'intimité et de solidarité tissés dans la parenté au sens large, il n'est pas rare d'observer des pressions de l'ordre de la sorcellerie vis-à-vis des élites qui se trouvent au village, dans l'administration, parmi les opérateurs privés, dans des projets ou des ONG. Des études devraient être menées sur cette question qui est probablement aussi importante que le patronage pour la compréhension de la coconstruction des rapports entre résidents locaux et acteurs de la gouvernance participative.

\section{La mobilisation des registres de sorcellerie}

Concernant le mécanisme participatif de l'allocation par mètre cube exploité de grume, les succès de la CEB en matière de développement rural sont en partie imputables à la «neutralisation" des élites. Les notables des villages puisent leur force mystiquement dans leur pouvoir traditionnel et résistent ainsi à l'influence des élites non résidentes.

La fonction de nivellement de la sorcellerie est également soulignée dans l'analyse de la relation entre résidents locaux et projet de conservation (Solly $2010: 177-193)$. Pour les Bulu, le développement est associé à l'inégalité, à la jalousie, ce qui provoque le déploiement d'une " sorcellerie niveleuse ", très répandue au sein de la communauté.

Des auteurs comme Fisiy \& Geschiere $(1993)$ et Geschiere $(1995,1996)$ ont analysé comment le rapport entre les nouvelles élites et le village débouche sur une nouvelle forme de sorcellerie, une "sorcellerie de la richesse ", qui révèle "une tension entre jalousie et ambition ».

«La sorcellerie est censée servir à la fois aux 'petits', comme arme égalisatrice contre les 'grands', et aux 'grands' eux-mêmes qui cherchent à consolider leur ascendant et à réaliser leurs ambitions » Geschiere (1996: 85).

Dans cette configuration, toute richesse attire la jalousie de ceux qui n'y ont pas accès et provoque des attaques de sorcellerie, tandis que l'origine suspecte des richesses accumulées place automatiquement l'élite en situation de sorcier.

Selon les auteurs d'analyses de la sorcellerie moderne contemporaine, il existe un lien étroit entre sorcellerie et corruption (voir par exemple Blundo \& Olivier de Sardan 2007). C'est à la faveur de sa position au croisement de la parenté et des rapports de pouvoir et de richesse que l'élite apparaît en mesure de détourner et d'accumuler en toute impunité, au profit des siens, les retombées financières en nature et symboliques de l'approche " participative».

En conséquence, plutôt que de passer par le canal d'une représentativité démocratique telle que conçue par le politique dans la décentralisation, les principes de la représentativité locale s'exercent selon les dynamiques redistributives en vigueur dans les pratiques corruptives et clientélistes ainsi que sous la contrainte d'une sorcellerie de la richesse. L'importance du phénomène de sorcellerie en zones forestières met aussi en exergue l'ancrage endogène des détournements de fonds destinés au développement local 
"participatif ». Dans des organisations sociales et politiques telles que celles du Bassin congolais, une part relativement importante des revenus paysans est ainsi investie dans des aides et prestations diverses afin d'entretenir un réseau de relations apte à protéger des effets niveleurs de la jalousie et à satisfaire des besoins souvent cruciaux de soins de santé, scolarité ou emploi.

Par ailleurs, toujours en raison de l'ampleur des pratiques de sorcellerie, les élites gardent une distance vis-à-vis du village. Elles n'investissent pas dans le développement de leur région, seulement dans leur propre développement, par exemple la construction d'une villa moderne ou d'une église en dur dans leur village d'origine.

Des dynamiques de cet ordre renseignent sur les logiques sous-jacentes aux stratégies locales vis-à-vis du développement "participatif». Bien que relevant, d'un côté, des morales de la corruption et, de l'autre, de l'éthos de la sorcellerie, ces pratiques sont complémentaires et ont le même caractère d'opacité du pouvoir et de la richesse, le même lien, la parenté, dans la relation entre village et élites, et la même dynamique entre intimité et espace public.

\section{Sécuriser le développement local}

Il est nécessaire de comprendre le poids de l'histoire coloniale qui pèse sur les expérimentations participatives dans le Bassin congolais pour analyser valablement les difficultés auxquelles ce mode de gouvernance est confronté. Le régime concessionnaire implanté dans cette région depuis la fin du XIX ${ }^{\mathrm{e}}$ siècle détermine encore des contraintes majeures, particulièrement en termes de recouvrements territoriaux, et limite considérablement la marge de manœuvre des acteurs de la gouvernance participative. Le contexte d'intervention du projet participatif, notamment de par ses particularités historiques et sociales et la primauté des relations de patronage, accroît les difficultés à atteindre des objectifs tangibles et durables dans les domaines de la démocratie locale et du développement participatif.

C'est dans un dispositif socio-historique similaire que les mécanismes participatifs sont expérimentés dans des secteurs aussi variés que l'exploitation du bois, la chasse et la conservation intégrée au développement rural, et ce sont des logiques et des stratégies locales également du même ordre qui peuvent être relevées. En conséquence, quel que soit le secteur concerné, c'est sur des recommandations similaires que débouche notre étude.

89 Au stade actuel, le mode de gouvernance participatif apparaît empreint de paternalisme, de laxisme, d'abus de pouvoir et de corruption. Fondé sur des dispositions légales et techniciennes occidentalo-centristes et néolibérales, il recourt peu aux autorités administratives et coutumières locales et va de pair avec une privation de terres et de ressources tout en dénotant un manque de coordination, un faible souci de sécurisation foncière et une non-reconnaissance du droit coutumier. Ce sont là autant de caractéristiques contraires aux valeurs du développement local participatif enracinées dans la démocratie, le respect des structures coutumières, la bonne gestion des fonds et la sécurisation juridique et financière.

Le fossé entre la rhétorique du projet " participatif » et les contraintes particulières de sa mise en œuvre constitue un premier niveau de difficultés à promouvoir des réalisations concrètes de développement local. Devant ces difficultés, qui peuvent être qualifiées de 
structurelles puisqu'elles relèvent du contexte postcolonial et clientéliste, il est nécessaire d'« adapter le contexte au dispositif participatif». Par exemple, ces politiques devraient être implantées dans des pays qui présentent un degré suffisant de liberté d'expression, d'abolition des rapports néocoloniaux et de contrôle des relations clientélistes.

91 La sécurisation de l'accès aux finages villageois devrait être une priorité de la gouvernance participative. Les plans de zonage nationaux ou régionaux devraient impérativement faire l'objet de déclassements ou de procédures de recouvrement légalement reconnues en faveur des communautés villageoises, dans une logique similaire à celle du droit de préemption, afin d'y insérer formellement les finages villageois.

$\mathrm{Vu}$ les nombreux repositionnements des acteurs locaux en réaction au dispositif de la gouvernance participative, il est nécessaire d'adopter un second niveau de mesures. Ces mesures « conjoncturelles » consistent, si possible, à « adapter le dispositif participatif au contexte ", par exemple en impliquant les instances décisionnelles coutumières ou en prenant en considération la dispersion géographique des groupes cibles. Cela permet d'éviter l'apparition de sous-comités de gestion vidés de tout pouvoir comme notre étude sur la redevance forestière annuelle communautaire l'a montré (Mbetoumou et al. 2010 : 57-81).

93 D'après la revue des mécanismes participatifs (Joiris \& Bigombe Logo 2010), la seule expérience plus ou moins porteuse de développement rural est celle de la CEB où l'opérateur privé gère les fonds et où des stratégies locales "gèlent » les rapports clientélistes. Il parait donc prioritaire de contrôler la gestion des ressources financières et de limiter l'emprise des élites dans la gestion des pouvoirs décentralisés afin d'améliorer le processus participatif dans le Bassin du Congo.

94 Compte tenu du caractère éclaté des microprojets de développement financés par les quatre filières "participatives", à savoir la fiscalité décentralisée, le secteur privé, l'entreprenariat local communautaire et le secteur classique de l'aide au développement, il parait nécessaire que les orientations données au développement local suivent des choix sectoriels définis et harmonisés au niveau macroéconomique.

Comme Diop (2006) dans son analyse de la décentralisation au Sénégal, nous proposons une meilleure articulation entre le niveau national et le niveau régional afin de pallier l'absence de communication entre collectivités locales; nous recommandons aussi d'établir des plans régionaux de développement intégré afin de mettre en place un " mécanisme national de coordination et d'harmonisation des différents programmes ».

Enfin, vu l'ampleur des stratégies locales déployées vis-à-vis des mécanismes participatifs, il serait judicieux de prévoir un suivi financier et technique. Cela semble d'autant plus urgent dans des situations où la réforme "participative » se solde par des pertes financières, en nature et en capital social. D'autres difficultés, en rapport surtout avec la faible capacité locale à exprimer des revendications foncières sur les terres d'avant la réforme "participative " et à s'opposer aux rapports omniprésents de domination et de clientélisme, indiquent clairement à quel point des évaluations régulières, des diagnostics en profondeur et des monitoring sont nécessaires pour mener à bien ce qui apparait comme un remaniement en profondeur des paysages politiques du Bassin congolais. 


\section{BIBLIOGRAPHIE}

Apthorpe R. 2011 - With Alice in Aidland: A seriously Satirical Allegory. In Mosse D. (Ed.). Adventures in Aidland: The Anthropology of Professionals in International Development. New York/ Oxford, UK, Berghahn Books : 199-219. (Studies in Public and Applied Anthropology).

Assembe S. 2004 - Justice environnementale, gestion durable et manipulation des revenus forestiers : une évaluation des impacts dans le Sud-Cameroun. Yaoundé, Cameroun, CIFOR, rapport de recherche non publié.

Béné C. \& Neiland A.E. 2006 - From Participation to Governance: A critical review of the concepts of governance, co-management and participation, and their implementation in small-scale inland fisheries in developing countries. Penang, Malaysia, The WorldFish Center, and Colombo, Sri Lanka, the CGIAR Challenge Program on Water and Food, 72 p. (WorldFish Center Studies and Reviews 29).

Bierschenk T. 2009 - Anthropologie et développement : Historiciser et localiser les approches. Bulletin de l'Apad (31-32) : 161-192.

Bigombe Logo P. 2002 - La gestion des conflits liés aux ressources naturelles. Cadre méthodologique. Yaoundé, Presses de l'UCAC, $30 \mathrm{p}$.

Bigombe Logo P. 2003a - Gérer autrement les conflits forestiers au Cameroun. Yaoundé, Presses de l'UCAC, $214 \mathrm{p}$.

Bigombe Logo P. 2003b - The decentralized forestry taxation system in Cameroon: local management and State logic. Washington DC, WRI, $35 \mathrm{p}$.

Bigombe Logo P. 2004 - Le retournement de l'État forestier. L'endroit et l'envers des processus de gestion forestière au Cameroun. Yaoundé, Presses de l'UCAC, 345 p.

Bigombe Logo P. \& Roulet P.-A. 2010 - L'évolution des zones d'intérêt cynégétique à gestion communautaire au Cameroun. In Joiris D.V. \& Bigombe Logo P. (Ed.). La gestion participative des forêts d'Afrique centrale. Un modèle à l'épreuve de la réalité. Versailles, QUAE : 143-151. (Synthèses).

Bigombe Logo P., Yamo A. \& Ngonde B. 2010 - Les forêts communautaires au village. In Joiris D.V. \& Bigombe Logo P. (Ed.). La gestion participative des forêts d'Afrique centrale. Un modèle à l'épreuve de la réalité. Versailles, QUAE : 105-119. (Synthèses).

Binot A., Joiris D. V. \& Hanon L. 2010 - L'échec de la sécurisation foncière dans les aires protégées. In Joiris D.V. \& Bigombe Logo P. (Ed.). La gestion participative des forêts d'Afrique centrale. Un modèle à l'épreuve de la réalité. Versailles, QUAE : 155-175. (Synthèses).

Blundo G. \& Olivier de Sardan J.-P. (Ed.) 2007 - État et corruption en Afrique. Une anthropologie des relations entre fonctionnaires et usagers (Bénin, Niger, Sénégal). Paris, APAD-Karthala, 375 p.

Borrini-Feyerabend G., Kothari A., Pimbert M.P., Renard Y. \& Taghi Farvar M. (Ed.) 2004 - Sharing Power. Learning by Doing in Co-management of Natural Resources Throughout the World. Teheran, IIEDUICN/CEESP/ CMWG, Centre for Sustainable Development and Environment, $456 \mathrm{p}$.

Cerutti P.-O., Lescuyer G., Assembe Mvondo S. \& Tacconi L. 2010a - Les défis de la redistribution des bénéfices monétaires tirés de la forêt pour les administrations locales : une décennie de redevance forestière assise sur la superficie du titre d'exploitation au Cameroun. Bogor, Indonesia, CIFOR, 15 p. (CIFOR Occasional Paper 53). 
Cerutti P.-O., Lescuyer G., Assembe Mvondo S. \& Tacconi L. 2010b - The challenges of redistributing forest-related monetary benefits to local governments: a decade of logging area fees in Cameroon. International Forestry Review 12 (2) : 130-138.

Cheka C. 2007 - The state of the process of decentralisation in Cameroon. Africa Development 33 (2) : 181-196.

Colfer C. \& Capistrano D. 2005 - Paths and pitfalls of decentralization for sustainable forest management: experiences of the Asia Pacific region. In Colfer C. \& Capistrano D. (Ed.). The politics of decentralization. Forests, power and people. London, Earthscan : 32-62.

Compagnon D. \& Constantin F. 2000 - Administrer l'environnement en Afrique. Gestion communautaire, conservation et développement durable. Paris, Karthala-Ifra, 497 p.

Cooke B. \& Kothari U. 2001 - Participation: The New Tyranny. Londres, Zed Books, 207 p.

Cuny P. 2011 - État des lieux de la foresterie communautaire et communale au Cameroun. Wageningen, Pays-Bas, Tropenbos International Programme du bassin du Congo, $110 \mathrm{p}$.

Cuny P., Abe'ele P., Nguenang G.M., Djeukam R., Eboule S. \& Eyene E. 2004 - État des lieux de la foresterie communautaire au Cameroun. Yaoundé, Ministère de l'Environnement et des Forêts, $114 \mathrm{p}$. De Jong W., Ruiz S. \& Becker M. 2006 - Conflicts and communal forest management in northern Bolivia. Forest Policy and Economics 8: 447-457.

Diaw M.C. 2005 - Modern Economy Theory and the Challenge of Embedded Tenure Institutions: African Attempts to Reform Local Forest Policies. In Kant S. \& Berry R.A. (Ed.). Sustainable Institutions and Natural Resources: Institutions for Sustainable Forest Management. Dordrecht, Springer Verlag : 43-83.

Diaw M.C., Assoumou H. \& Dikongué E. 1998 - Anthropological Institutions and Forest Management. What Institutional Framework for Community-Based Forest Management in Cameroon? Bulletin FTPP $13: 20-25$.

Diop D. 2006 - Décentralisation et gouvernance locale au Sénégal. Quelle pertinence pour le développement local ? Paris, L'Harmattan, $267 \mathrm{p}$.

Douglas M. 1986 - How Institutions Think. Syracus, New York, Syracus University Press, 128 p. Douglas M. 2004 (1999) - Comment pensent les institutions. Suivi de La connaissance de soi et Il n'y a pas de don gratuit. Paris, La Découverte, $218 \mathrm{p}$.

Dounias E. 1993 - Dynamique et gestion différentielles du système de production à dominante agricole chez les Mvae du Sud-Cameroun forestier. Thèse de doctorat, Physiologie, Université de Montpellier II, $490 \mathrm{p}$.

Dounias E.,2000 - La diversité des agricultures itinérantes sur brûlis. In Bahuchet S. \& Maret P. de (Ed.). Avenir des peuples des forêts tropicales, Approche thématique. Bruxelles, Commission européenne, direction générale VIII : 65-106.

Emerit A. \& Lescuyer G. 2003 - Trois étapes pour la gestion viable de la forêt camerounaise : comprendre, énoncer, concilier les logiques d'acteurs. Sociologies Pratiques 7 : 29-48.

Etoungou P. 2003 - Decentralization viewed from inside: the implementation of community forests in east Cameroon. Washington DC, USA, WRI : 28 p.

Ezzine de Blas D., Ruiz Pérez M., Sayer J.A., Lescuyer G., Nasi R. \& Karsenty A. 2009 - External Influences on and Conditions for Community Logging Management in Cameroon. World development 37 (2) : 445-456. 
Ezzine de Blas D., Ruiz-Pérez M. \& Vermeulen M. 2011 - Management conflicts in Cameroonian c ommunity forests. Ecology and Society 16 (1): 8 [En ligne] URL: http://www.ecologyandsociety.org/ vol16/ iss1/art8/. Consulté le 13 février 2014.

Ferguson J. 1994 - The Anti-Politics Machine: Development, De-politicisation and Bureaucratic Power in Lesotho. Minneapolis, University Minneapolis Press, 323 p.

Ferguson J. 2005 - Seeing like an oil company: space, security and global capital in neoliberal Africa. American Anthropologist 107 : 377-82.

Ferguson J. 2006 - Global Shadows: Africa in the Neoliberal World Order. Durham, London, Duke University Press, 263 p.

Ferguson J. 2010 - The uses of neoliberalism. Antipode 41 (Suppl. 1) : 166-84.

Ferguson J. \& Gupta A. 2002 - Spatializing states: towards an ethnography of neoliberal governmentality. American Ethnologist 29 : 981-1002.

Fisiy C.F. \& Geschiere P. 1993 - Sorcellerie et accumulation, variations régionales. In Geschiere P. \& Konings P. (Ed.). Itinéraires d'accumulation au Cameroun. Paris, Karthala : 99-130. (Hommes et société).

Garcia C.-A. \& Lescuyer G. 2008 - Monitoring Indicators and Community-Based Forest Management in the Tropics: Pretexts or Red Herrings? Biodiversity and Conservation 17 (6) : 1303-1317.

Garnett S., Sayer J. \& Du Toit J. 2007 - Improving the effectiveness of interventions to balance conservation and development: a conceptual framework. Ecology and Society 12 (1), [En ligne], http://www.ecologyandsociety.org/vol12/iss1/art2/. Consulté le 14 février 2014.

Geschiere P. 1995 - Sorcellerie et politique en Afrique. La viande des autres. Paris, Karthala, 300 p. Geschiere P. 1996 - Sorcellerie et politique : Les pièges du rapport élite-village. Politique africaine $63: 82-96$.

Geschiere P. 2004 - Ecology, belonging and xenophobia: the 1994 forest law and the issue of « community ». In England H. \& Nyamnjoh F. (Ed.). Rights and the politics of recognition in Africa. London, Zed Books : 237-261.

Graig D. \& Porter D. 2006 - Development Beyond Neoliberalism? Governance, Poverty Reduction and Poltical Economy. London, New York, Routledge, 340 p.

Gudeman S. 2001 - The Anthropology of Economy: Community, Market and Culture. Malden, Oxford, Blackwell Publishers, 189 p.

Hickey S. \& Mohan G. 2004 - Participation: From Tyranny to Transformation? London, Zed, 277 p. Igoe J. \& Brockington D. 2007 - Neoliberal Conservation: A Brief Introduction. Conservation and Society 5 (4) : 432-449.

IIED 1994 - Whose Eden? An Overview of Community Approaches to Wildlife Management. Londres, IIED, $124 \mathrm{p}$.

Isaacman A. 1997 - Historical amnesia or the logic of capital accumulation: cotton production in colonial and postcolonial Mozambique. Environment and Planning D: Society and Space 15: 757-790.

Iversen V., Chettry B., Francis P., Gurung M., Kafle G., Pain A. \& Seeley J. 2006 - High value forests, hidden economies and elite capture: evidence from forest user groups in Nepal's Terai. Ecological Economics 58 : 93-107. 
Joiris D.V. \& Bigombe Logo P. (Ed.) 2010 - La gestion participative des forêts d'Afrique centrale. Un modèle à l'épreuve de la réalité. Versailles, QUAE, 236 p. (Synthèses).

Joiris D.V. \& Bigombe Logo P. 2012 - L'heuristique de la littérature grise sur le développement participatif du Bassin congolais. Bulletin de l'Apad 34-36 : 217-235.

Julve C., Vandenhaute M., Vermeulen C., Castadot B., Ekodeck H. \& Delvingt W. 2007 - Séduisante théorie, douloureuse pratique : la foresterie communautaire camerounaise en butte à sa propre législation. Parcs et Réserves 62 (2) : 18-24.

Kapos V., Balmford A., Aveling R., Bubb P., Carey P., Entwistle A., Hopkins J., Mulliken T., Safford R., Stattersfield A., Walpole M. \& Manica A. 2009 - Outcomes, not implementation, predict conservation success. Oryx 43 (3) : 336-342.

Karsenty A. 2006 - L'impact des réformes dans le secteur forestier en Afrique centrale. In Nasi R., Nguinguiri J.-C. \& Ezzine-de-Blas D. (Ed.). Exploitation et gestion durable des forêts en Afrique Centrale. Paris, L'Harmattan : 25-60.

Karsenty A. 2008 - Des communautés locales problématiques. In Méral P., Castellanet C., Lapeyre R. (Dir.). La gestion concertée des ressources naturelles. L'épreuve du temps. Gret-Karthala : 277-288.

Karsenty A. 2010 - La responsabilité sociale et environnementale des entreprises concessionnaires. In Joiris D.V. \& Bigombe Logo P. (Ed.). La gestion participative des forêts d'Afrique centrale. Un modèle à l'épreuve de la réalité. Versailles, QUAE : 35-53. (Synthèses).

Kiss A. (Ed.) 1990 - Living with Wildlife: Wildlife Resource Management with Local Participation in Africa. Washington, Banque mondiale, $217 \mathrm{p}$.

Kumar S. 2002 - Does « participation » in common pool resource management help the poor? A social cost-benefit analysis of joint forest management in Jharkhand, India. World Development 30 (5) : 763-782.

Kupper A. 2003 - The return of the Native. Current Anthropology 44 (3) : 389-402.

Larson A.M. 2005 - Democratic decentralization in the forestry sector : lessons learnt from Africa, Asia and Latin America. In Colfer C. \& Capistrano D. (Ed.). The politics of decentralization. Forests, power and people. London, Earthscan : 32-62.

Larson A.M., Barry D. \& Ram Dahal D. 2010 - New rights for forest-based communities ? Understanding processes of forest tenure reform. International Forestry Review 12 (1) : 78-96.

Lavigne Delville P. 1992 - Participation paysanne, discours et pratiques. Quelques réflexions sur le texte de J.-P. Chauveau. Bulletin de l'Apad 3, [En ligne], URL : http://apad.revues.org/381. Consulté le 14 février 2014.

Lavigne Delville P., Mathieu M., Sellamna N. (Ed.) 2000 - Les enquêtes participatives en débat. Ambition, pratique et enjeux. Paris-Montpellier, Karthala- Gret-Icra, 543 p.

Lescuyer G. 2006 - Formes d'action collective pour la gestion locale de la forêt camerounaise : organisations 'modernes' ou institutions 'traditionnelles' ? Vertigo 6 (3), [En ligne] URL : http:// vertigo.revues.org/index8029.html. Consulté le 6 avril 2009.

Lewis D. 2009 - Anthropology, Development and the 'Perpetual Present'. Knowledge, Power and Practice. In Hagberg S. \& C. Widmark (Ed.). The ethnographic practice and public aid. Methods and meanings in development cooperation. Uppsala Studies in Cultural Anthropology 45: 53-71.

Lewis D. \& Mosse D. (Ed.) 2006 - Development Brokers and Translators: The Ethnography of Aid and Agencies. Bloomfield, Kumarian Press, 251 p. 
Li T.M. 2002 - Engaging simplifications: community-based resource management, market processes and states agendas in Upland Southeast Asia. World Development $30: 265-283$.

Li T.M. 2007 - The Will to Improve: Governmentality, Development, and the Practice of Politics. Durham, NC, Duke University Press, 375 p.

Lockwood M., Davidson J.-L., Curtis J., Stratford E., Griffith R. 2010 - Governance principles for natural resource management. Society and Natural Resources, 23 (10) : 986-1001.

Long N. \& Long A. (Ed.) 1992 - Battlefields of Knowledge: The Interlocking of Theory and Practice in Social Research and Development. London, New York, Routledge, 306 p.

Mbetoumou M., Joiris D. V. \& Abega S. C. 2010 - La gestion par le haut de la redevance forestière annuelle communautaire. In Joiris D.V. \& Bigombe Logo P. (Ed.). La gestion participative des forêts d'Afrique centrale. Un modèle à l'épreuve de la réalité. Versailles, QUAE : 57-81. (Synthèses).

Méral P., Castellanet C. \& Lapeyre R. 2008 - La gestion concertée des ressources naturelles. L'épreuve du temps. Paris, Gret-Karthala, 333 p.

Mosse D. 2005 - Cultivating Development: An Ethnography of Aid Policy and Practice. London, Pluto, $315 \mathrm{p}$.

Mosse D. (Ed.) 2011a - Adventures in Aidland: The Anthropology of Professionals in International Development. New York/Oxford UK, Berghahn Books, 238 p. (Studies in Public and Applied Anthropology).

Mosse D. 2011b - Introduction: The Anthropology of Expertise and Professionals in International Development. In Mosse (Ed.). Adventures in Aidland: The Anthropology of Professionals in International Development. New York/Oxford UK, Berghahn Books : 1-33.

Mosse D., 2013 - The Anthropology of International Development. Annual Review of Anthropology $42: 227-46$

Nasi R., Nguiguiri J.-C. \& Ezzine de Blas D. (Ed.) 2006 - Exploitation et gestion durable des forêts en Afrique centrale. Paris, L'Harmattan, $404 \mathrm{p}$.

Nelson F. \& Agrawal A. 2008 - Patronage or Participation? Community-Based Natural Resource Management Reform in Sub-Saharan Africa. Development and Change 39 : 557-585.

Ngo Youmba F.-M. 2007 - « Manger la forêt ». Jè lipan, ou la construction sociale de la foresterie en pays Babimbi au sud du Cameroun. Stuttgart, Ibidem, $312 \mathrm{p}$.

Nguimbi L., Roulet P.-A. \& Nzang Oyono C. 2010 - La gestion locale assumée par un opérateur privé. In Joiris D.V. \& Bigombe Logo P. (Ed.). La gestion participative des forêts d'Afrique centrale. Un modèle à l'épreuve de la réalité. Versailles, QUAE : 86-102. (Synthèses).

Nguinguiri J.-C. \& Pierre J.-M. 2006 - La durabilité sociale de la gestion des concessions forestières dans le Bassin du Congo : faut-il réinventer la roue ? In Nasi R., Nguinguiri J.-C. \& Ezzine de Blas D. (Ed.). Exploitation et gestion durable des forêts en Afrique centrale. La quête de la durabilité. Paris, L'Harmattan : 299-316.

Olivier de Sardan J.-P. 1995 - Anthropologie et développement : essai en socio-anthropologie du changement social. Paris, Apad-Karthala, 222 p.

Oyono P.R. 2005a - Profiling Local-Level Outcomes of Environmental Decentralizations: The Case of Cameroon's Forests in the Congo Basin. Journal of Environment and Development 14 (2) : 1-21.

Oyono P.R. 2005b - The foundations of the conflit de langage over land and forests in southern Cameroon. African Study Monographs 26 (3) : 115-144. 
Oyono P.R. 2006 - Acteurs locaux, représentation et 'politics' des éco-pouvoirs dans le Cameroun rural post-1994. Canadian Journal of Development Studies 27 (2) : 163-185.

Oyono P.R. \& Efoua S. 2006 - Qui représente qui ? Choix organisationnels, identités sociales et formation d'une élite forestière au Cameroun. Afrique et développement 31 (2) : 147-182.

Oyono P.R., Diaw M.-C. \& Sangkwa F. 2006a - Les réformes forestières et les nouvelles frontières de la gestion locale au Cameroun. In Nasi R., Nguinguiri J.-C. \& Ezzine de Blas D. (Ed.). Exploitation et gestion durable des forêts en Afrique centrale. La quête de la durabilité. Paris, L'Harmattan : 317-367.

Oyono P.R., Ribot J.C. \& Larson A. M. 2006b - Green and black gold in rural Cameroon: natural resources for local governance, justice and sustainability. Washington DC, USA, WRI, $92 \mathrm{p}$.

Oyono P.R., Larson A.-M. \& Ribot J. 2007a - Or vert et or noir dans le Cameroun rural. Washington D.C., WRI, 96 p.

Oyono P.R., Ribot J. C., Assembe S. \& Bigombe Logo P. 2007b - Correctifs pour la Gestion Décentralisée des Forêts au Cameroun : Options et Opportunités de Dix Ans d'Expérience. Bogor, Indonesia, CIFOR, 12 p. (CIFOR Brief ; 33).

Pacheco P. 2005 - Decentralization of forest management in Bolivia: who benefits and why? In Colfer C. \& Capistrano D. (Ed.). The politics of decentralization. Forests, power and people. London, UK, Earthscan : 168-183.

Pigg S.L. 1992 - Inventing Social Categories through Place: Social Representation and Development in Nepal. Comparative Study in Society and History 34 : 491-513.

Rajak D. 2011 - In Good Company: An Anatomy of Corporate Social Responsibility. Stanford, California, Stanford University Press, $320 \mathrm{p}$.

Ribot J.C. 2004a - La décentralisation démocratique des ressources naturelles. Institutionnaliser la participation populaire. Washington DC, USA, WRI, $34 \mathrm{p}$.

Ribot J.C. 2004b - Waiting for Democracy: The Politics of Choice in Natural Resource Decentralisation. Washington DC, WRI, $142 \mathrm{p}$.

Ribot J.C. 2008 - Non-décentralisation démocratique au Sénégal : le non-transfert de l'autorité sur les forêts. Washington DC, USA, WRI, $34 \mathrm{p}$.

Ribot J.C., Agrawal A. \& Larson A. M. 2006 - Recentralizing while decentralizing: how national governments reappropriate forest resources. World Development 34 (11):1864-1886.

Robbins P. 2000 - The rotten institution: corruption in natural resource management. Political Geography 19 : 423-443.

Rodary E. 2001 - Les espaces naturels : l'aménagement par la participation? Mise en réseau et territorialisation des politiques de conservation de la faune en Zambie et au Zimbabwe. Thèse de doctorat, Géographie, Université d'Orléans, Orléans, 531 p.

Rossi B. 2004a - Revisiting Foucauldian Approaches : Power Dynamics in Development Projects. Journal of Development Studies 40 :1-29.

Rossi B. 2004b - Order and Disjuncture: Theoretical Shifts in the Anthropology of Aid and Development. Current Anthropology 45 (4) : 556-560.

Rossi B. 2006 - Aid Policies and Recipient Strategies in Niger. In Lewis D. \& Mosse D. (Ed.) Development Brokers and Translators: The Ethnography of Aid and Development. Kumarian, Bloomfield : 27-49. 
Rossi B. 2007 - Les Paradoxes des Aides Chroniques. In Crombé X. \& Jézéquel J.H. (Ed.) Niger 2005 : Une catastrophe si naturelle. Paris, Karthala : 145-172.

Roulet P.-A. \& Hardin R. 2010 - Des domaines de chasse aux zones d'intérêt cynégétique à gestion communautaire. In Joiris D.V. \& Bigombe Logo P. (Ed.). La gestion participative des forêts d'Afrique centrale. Un modèle à l'épreuve de la réalité. Versailles, QUAE : 123-139. (Synthèses).

Sayer J., Campbell B., Petheram L., Aldrich M., Ruiz-Perez M., Endamana D., Dongmo Z. \& Defo L. 2007 - Assessing environment and development outcomes in conservation landscapes.

Biodiversity and Conservation 16 (9) : 2677-2694.

Schwartz N.B. 1981 - Anthropological views of community and community development. Human Organization 40 (4) : 313-322.

Sindjoun L. 1998 - La politique d'affection en Afrique noire. Boston, Boston University (Groupe de recherche sur l'Afrique francophone de l'African Studies Center), $64 \mathrm{p}$.

Siswanto W. \& Wardojo W. 2005 - Decentralization of the forestry sector: Indonesian's experience. In Colfer C. \& Capistrano D. (Ed.). The politics of decentralization. Forests, power and people. London, UK, Earthscan : 141-151.

Sletto B. I. 2009 - 'We Drew What We Imagined'. Participatory Mapping, Performance, and the Arts of Landscape Making. Current Anthropology 50 (4) : 443-476.

Solly H. 2010 - Donner, recevoir ou prendre dans la réserve du Dja au Cameroun. In Joiris D.V. \& Bigombe Logo P. (Ed.). La gestion participative des forêts d'Afrique centrale. Un modèle à l'épreuve de la réalité. Versailles, QUAE : 177-193. (Synthèses).

Sommerville M., Jones J., Rahajaharison M. \& Milner-Gulland E. J. 2010 - The role of fairness and benefit distribution in community-based payment for environmental services interventions: a case study from Menabe, Madagascar. Ecological Economics 69 : 1262-1271.

Sunderland T.C.H., Sayer J. \& Hoang M.-H. (Ed.) 2012 - Evidence-based Conservation. Lessons from the Lower Mekong. New York, Routledge, 480 p. (The Earthscan Forest Library).

Sundström A. 2013 - Corruption in the commons: Why briebery hampers enforcement of environmental regulations in South African fisheries? International Journal of the Commons 7 (2) : 454-472.

Tacconi L. 2007 - Decentralization, forests and livelihoods: theory and narrative. Global Environmental Change 17 : 338-348.

Tsing A.L. 1999 - Becoming a tribal elder and other green development fantasies. In Li T.M. (Ed.) Transforming the Indonesian Uplands. London \& New York, Routledge : 157-201. (Studies in Environmental Anthropology).

Turner M.D. 1999 - Conflict, environmental change, and social institutions in dryland Africa : limitation of the community resource management approach. Society and Natural Resources 12 : 643-658.

Uphoff N. 1998 - Learning about and for Participation. From Theoretical and Empirical Studies to Practical Experience and back to Theory. Canadian Journal of Development Studies19 (3) : 437-460.

Vansina J. 1991 - Sur les sentiers du passé en forêt. Les cheminements de la tradition politique ancienne de l'Afrique équatoriale. Louvain-la-Neuve, Université catholique de Louvain (Centre d'histoire de l'Afrique), 407 p. (Enquêtes et documents d'histoire africaine ; 9).

Vermeulen C., Vandenhaute M., Dethier M., Ekodeck H., Nguenang G.-M. \& Delvingt W. 2006 - De Kompia à Djolempoum : sur les sentiers tortueux de l'aménagement et de l'exploitation des forêts 
communautaires au Cameroun. Vertigo 7 (1), [En ligne], Volume URL : http:// vertigo.revues.org/2149; DOI : 10.4000/vertigo.2149. Consulté le 14 février 2014.

Wade R. 1988 - Village republics: economic conditions for collective action in South India. Cambridge, New York, Cambridge University Press, 238 p.

Wade R. 1996 - Japan, the World Bank and the Art of Paradigme Maintenance. New Left Review 217 : 3-36.

Weber J. 2000 - Pour une gestion sociale des ressources naturelles. In Compagnon D. \& Constantin F. (Ed.). Administrer l'environnement en Afrique. Gestion communautaire, conservation et développement durable. Paris-Nairobi, Karthala-Ifra : 79-105.

Wells M., Brandon K. \& Hannah L. 1992 - People and Parks : Linking Protected Area Management with Local Communities. Washington, World Bank-WWF- USAID, 99 p.

White S.C. 1996 - Depoliticizing Development : The Uses and Abuses of Participation. Development in Practice 6 (1) : 6-15.

Wollenberg E. 1998 - A conceptual framework and typology for explaining the outcomes of local forest management. Journal of World Forest Resource Management 9 : 1-35.

\section{NOTES}

1. L'article a été écrit avec la collaboration d'Aurélie Binot, Chimère Mariteuw Diaw, Laurence Hanon, Rebecca Hardin, Alain Karsenty, Marceline Mbetoumou, Léon Nguimbi, Benjamen Ngonde, Claire Nzang Oyono, Pierre-Armand Roulet, Hilary Solly, Jacques Weber et Antang Yamo. 2. Ainsi que sa propre ethnographie de la politique de l'aide en Inde (Mosse, 2005) et des auteurs comme Pigg (1992) pour le Népal, Li (2007) pour l'Indonésie, Rossi (2004a, 2004b) pour le Niger.

3. Rendering technical au sens de $\mathrm{Li}$ (2007)

4. Notre traduction.

5. Tiré de l'ouvrage édité en 2010 aux éditions Quae dans la collection Synthèses «Gestion participative des forêts d'Afrique centrale. Un modèle à l'épreuve de la réalité »

6. Au Cameroun, le montant global de plus de 47 milliards de francs CFA versé aux communes rurales et aux populations riveraines des forêts soumises à exploitation, au titre de la rétrocession de $50 \%$ de la redevance forestière annuelle (RFA), de 2000 à fin 2007, n'a pas été investi dans le développement.

7. Integrated Conservation and Development via des dispositifs participatifs.

8. Sur cette vison essentialiste, idéalisée et simplificatrice des " groupes cibles » de l'aide, voir Li (2002), voir Olivier de Sardan (1995), Schwartz (1981) et Karsenty (2008).

9. Sur l'aspect « dépolitisé » ou « anti-politique » de l'offre de développement, voir White (1996) dans la veine de Ferguson (1994), ainsi que Ribot et al. 2006.

\section{RÉSUMÉS}

La gestion participative des forêts est expérimentée dans le Bassin congolais depuis une trentaine d'années. Mais, ses résultats sont mitigés. Cette préoccupation constitue la base de cette 
réflexion. Les auteurs soulignent la complexité des mécanismes sous-jacents aux difficultés identifiées et entreprennent leur analyse d'un triple point de vue : celui du fonctionnement développementiste, celui du contexte socio-politique et celui des rapports de force en présence. S'ancrant dans une démarche à la fois historique et ethnographique, ils discutent la performance de quatre filières de financement "participatives" (fiscalité décentralisée, secteur privé, entreprenariat communautaire local, secteur classique de l'aide au développement) et de cinq mécanismes participatifs (rente forestière annuelle communautaire, rente de superficie, forêt communautaire, zone d'intérêt cynégétique à gestion communautaire, activités de conservation et de développement intégrées). A partir d'une analyse comparative trans-sectorielle, ils mettent en évidence que cette politique relève globalement d'une gouvernance pratique antiparticipative qui n'invalide pas le modèle participatif en soi, mais remet en cause la manière dont il est mis en œuvre. L'article se termine sur l'impératif d'une bonne connaissance préalable des contextes de réalisation des approches participatives et du monitoring des pratiques empiriques de la gestion participative et du développement rural effectif.

Participatory management, like any policy, can be considered as a concept, a policy practice and an actors-oriented issue. The poor results found in the Congolese Basin where this approach to rural development is experienced over the past thirty years are discussed in this article. The authors emphasize on the complexity of the mechanisms underlying the difficulties identified and begin their analysis with a triple point of view: that of the aid organization, the sociopolitical context and the power relations involved. From a historical and ethnographic point of view, they discuss the performance of four "participatory " funding and five participatory mechanisms. Based on a cross-sectoral analysis, they show that this policy is generally a antiparticipative governance. The article ends with the imperative of good knowledge on social contexts of implementation of participative approaches and monitoring empirical practices of participative management and of an effective rural development.

\section{INDEX}

Mots-clés : gestion participative, ressources naturelles, fiscalité décentralisée, secteur privé, forêt communautaire, zone d'intérêt cynégétique à gestion communautaire, développement intégré à la conservation

Index géographique : Afrique centrale

Keywords : participatory management, natural resources, decentralized taxation, private sector, community forest, community hunting area, integrated conservation and development

\section{AUTEURS}

\section{DAOU VÉRONIQUE JOIRIS}

Docteur en anthropologie sociale, consultante spécialisée dans les relations entre nature et société, chargée de cours à l' Université Libre de Bruxelles, Centre d'Anthropologie Culturelle, Institut de Sociologie, Belgique
44, Avenue Jeanne
1050 Bruxelles
Belgique
Tel. : 003226503428
Fax. : 003226504337
vjoiris@ulb.ac.be 


\section{PATRICE BIGOMBE LOGO}

Politologue, enseignant-chercheur au Groupe de recherches administratives, politiques et sociales (GRAPS) de l'Université de Yaoundé II et à la Fondation Paul Ango Ela pour la géopolitique en Afrique centrale, directeur du Centre de recherche et d'action pour le développement durable en Afrique centrale (CERAD), Yaoundé, Cameroun

Tel. : 0023777758310

patricebigombe@hotmail.com

\section{SÉVERIN CÉCILE ABEGA (†)}

Docteur en anthropologie, professeur à la faculté des sciences sociales et de gestion de l'université catholique d'Afrique centrale (UCAC), Institut catholique de Yaoundé, directeur de l'Institut des recherches socio-anthropologiques (IRSA), Yaoundé, Cameroun 